\title{
AUTOMATIC GENERATION OF ANCIENT POTTERY PROFILES USING CAD SOFTWARE
}

\begin{abstract}
The paper presents a new approach for the virtual reconstruction of historical artifacts such as ceramic pots. The technique is based on the photogrammetry method through which the profile line of the pot is achieved and which is composed by a fixed number of inflection points. The parameterization of the profile is then followed by the creation of a macro (script) in CAD software in order to automatically generate the shape of the ceramic pot. The case study included in the article consists of a total of 15 families of ancient ceramic pottery. The purpose of this technique is to serve as a support both for historians, archaeologists and restorers, as well as for students who desire a fast, fair and accessible virtual reconstruction of ceramic pots, regardless of their considered knowledge in designing or editing a programming language.
\end{abstract}

Keywords: ancient pottery; virtual reconstruction; spline curve; inflexion point; macro programming.

\section{INTRODUCTION}

s early as ancient times, our ancestors have created handmade objects in order to facilitate their everyday life. Ceramic objects fall into this rategory and have been used over the years in various daily activities, including those of religious kind ${ }^{1}$.

The pottery recovered during archaeological excavations is, above all, a historical and artistic document, and in terms of numerical ratio it is found first among the categories of materials subject to restorations of historical artifacts.

Reconstruction of ceramic artifacts from fragments found in archaeological sites represents a tedious task that requires many hours of work on behalf of restoration personnel, curators and archaeologists. The reason for such a thing is that ceramic fragments found in archaeological sites may be related to different pots or other objects that may have different shapes and colors. In addition, in recent years the tendency to digitize cultural heritage is continuously increasing. This is largely due to the actions taken by the European Union through the creation of various projects that encourage such an endeavor. It is now well known that the EU is supportive of the leading research efforts regarding the development of new techniques and methodologies for cultural heritage preservation.

As a response to such factors, many computer graphics researchers are focused on creating different methods and algorithms that can automatically reconstruct pottery using information extracted from the existing fragments. This information highlights and compares many features of the ceramic pot including its shape, edges and color. Such a research was developed by Igbal Marie and Hisham Qasrawi from the Department of Civil Engineering of The

\footnotetext{
1 GHEORGHIU 2005, 138-146.
}

\section{Ionuț Badiu}

Technical University of Cluj-Napoca ionut.badiu@muri.utcluj.ro

\section{Zsolt Buna}

Technical University of Cluj-Napoca zsolt.buna@muri.utcluj.ro

\section{Radu Comes}

Technical University of Cluj-Napoca radu.comes@muri.utcluj.ro

DOI: $10.14795 /$ j.v2i2.110

ISSN $2360-266 \mathrm{X}$

ISSN-L 2360 - 266X 
Hashemite University and it consists of a non-destructive optical technique that makes use of the 3D MAX modeling software in order to obtain the missing pieces and virtually assemble the ceramic pots. The matching of fragments and their alignment is based on the virtual matching of profiles and edges of the broken parts. This technique assists the archaeologists in reconstructing the shape of the pottery, while providing a useful estimation of valid fragments combinations ${ }^{2}$.

Also, computer graphic researchers Martin Kampel and Robert Sablatning from the Institute for Computer Aided Automation from Technical University of Vienna have developed a system that can be applied to both complete and broken pots using two different reconstruction strategies. For fragments, the shape of the profile is created using the profile method. The profile represents the cross-section of the fragment in the direction of the rotation axis of symmetry and its shape is a closed curve in the plane. For complete pottery, the $3 \mathrm{D}$ reconstruction is based on a sequence of images of the same object, but taken from different viewpoints. Both researches use algorithms to construct the 3D model of the pot for classification and display ${ }^{3}$.

The development of content-based retrieval mechanism that can extract information from pottery fragments represents a very active research area, thus this paper proposes a methodology that can automatically reconstruct ceramic pottery using information extracted from existing fragments and profile drawings. The method makes use of Visual Basic Script and Macro Programming in order to automatically create the pottery profiles in 3D. Also, it greatly simplifies the modeling process.

\section{RECONSTRUCTION TECHNIQUES FOR HISTORICAL ARTIFACTS SUCH AS CERAMIC POTTERY}

It is known that, nowadays, the reconstruction of ceramic pots recovered from archaeological sites is achieved by two different procedures: a classical one consisting of the manual reconstruction of the artifact ${ }^{4}$, and a virtual one, in which such reconstruction is computer-aided ${ }^{5}$. In turn, the virtual reconstruction process is divided into two different methods: the photogrammetry method and the virtual reconstruction method based on laser scanning.

The specialized magazine named Photogrammetric Engineering and Remote Sensing defines photogrammetry as being "the art, science and technology of obtaining reliable information about physical objects and the environment by processing records, measuring and interpreting photographic images, patterns of electromagnetic radiant

\footnotetext{
2 MARIE/QASRAWI 2005, 1527-1533.

3 KAMPEL/SABLATNIG 2003, 318-325.

4 CURSARU HERLEA 2008, 249-262.
}

LIU/POTTMANN/WANG 2006, 572-583; OXHOLM/ NISHINO 2013, 51-61; KARASIK/SMILANSKY 2008, 11481168; GILBOA/TAL/SHIMSHONI/KOLOMENKIN 2013, 13291339; LAPP/NICOLI 2014, 34-44; WEISE/WISMER/LEIBE/ VAN GOOL 2011, 635-648; BRUNO/BRUNO/DE SENSI/LUCHI/ MANCUSO/MUZZUPAPPA 2010, 42-49; CHOW/CHAN 2009, 161-173; COHEN/LIU/EZGI 2013，41-53; SMITH/KARASIK/ NARAYANAN/OLSON/SMILANSKY/LEVY 2014, 212-250; ITSKOVICH/TAL 2011, 334-341; MONTES/MELERO 2010, 355358. energy and other phenomena". In other words, one may say that photogrammetry is the science and technology that is mainly aimed at obtaining basic data in the form of photograms (as they fulfill certain conditions, in particular metric conditions), recognition and identification of object records, the determination of their shape and size, and the materialization of such results in analog and/or digital form. In short, photogrammetry is the science of determining measurements by photography.

This method of reconstruction is relatively simple, it has a low cost and it can be used as well for objects consisting of a high degree of complexity, but because it is based on photographs, it causes the need for adequate space ${ }^{6}$ in terms of ambient light and clarity of captured photos.

A major advantage of this method is the brief time spent with the three-dimensional digital model generation. 3D modeling technique based on images shows the usefulness of digital photogrammetry by an accurate modeling and visualization in a virtual space of real objects defined by regular geometric shapes. The precision by which 3D models are obtained (within one pixel) with such a procedure corresponds to the reconstruction applications of historical heritage conservation, photogrammetry thus being the best alternative to conventional measurement techniques ${ }^{7}$. Another important characteristic for imagebased reconstructions is that the ceramic pottery is not being touched during the process. This is a requirement commonly seen in fragile artifacts.

Furthermore, due to recent research in the field of photogrammetry, the accuracy of this method has increased, making it a viable solution for creating the $3 \mathrm{D}$ digital content of the cultural heritage ${ }^{8}$.

In order to validate this method, the authors of this paper have conducted a digitization example of a ceramic pot by means of the Autodesk Memento software. The principle of such a procedure lays in the automatic identification of a set of points corresponding to sequential images taken on two or more images that cover the same portion of the artifact. Thus, by means of photogrammetry principles, the $3 \mathrm{D}$ coordinates for the virtual processing of the ceramic pot are extracted ${ }^{9}$.

The digitized pot obtained after processing the images is shown in figure 1.

The process of reconstruction by means of photogrammetry is also used in combination with laser scanning, in order to face

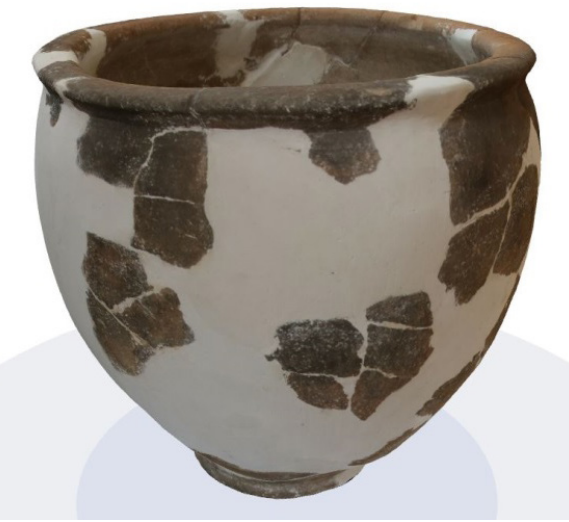

Fig. 1. A digitized ceramic pot obtained through photogrammetry.

6 PAVLIDIS/KOUTSOUDIS/ARNAOUTOGLOU/TSIOUKAS/ CHAMZAS 2007, 93-98.

7 VILCEANU 2013.

8 COMES/BUNA/BADIU 2014, 50-57.

9 KOUTSOUDIS/ARNAOUTOGLOU/GHAMZAS 2007, 26-31. 
the challenges raised by the resolution, accuracy and operational requirements regarding the digitization of historical artifacts. The reason for this is that photogrammetry is less accurate than laser scanning, since it is based, most of the time, on a hand drawing or on the picture of the pot ${ }^{10}$. In other words, photogrammetry is a rapid measurement technique that produces models through the use of information from colors, light and laser scanning, by perfectly capturing the 3D geometry of any object and automatically obtaining a high resolution result. Yet, the above statement excludes the objects that reflect light or are transparent; therefore, a combination of both photogrammetry and laser scanning is undoubtedly beneficial ${ }^{11}$.

\section{CASE STUDY ON THE GENERATION OF CERAMIC POTTERY FAMILIES STARTING FROM THE PROPOSED METHODOLOGY}

Based on the process of reconstruction by photogrammetry, the authors of this paper have developed a new approach to virtual reconstruction of historical artifacts such as ceramic pots, starting from the parameterization of the pot's profile through a fixed number of inflection points. This crucial first step is in fact the input information for the second part of the proposed methodology in which, by means of a script provided in a CAD software, the automatic generation of the pot takes place.

The case study in this paper is comprised of a total of 15 families of ceramic pots generated with the help of CATIA V5R21 design software, using photogrammetry as a method of reconstruction.

The key to virtual reconstructions of pottery using this method is the establishment of the profile line ${ }^{12}$. This is done after the insertion of the pot's image at 1:1 scale in the design software (in this case, CATIA V5R21). After the insertion, a sketch is created on the front plane of the inserted image, followed by the drawing of the profile line using eight points of inflection contained by the spline curve (Figure 2).

Each inflection point of the profile curve is defined by two pairs of coordinates, as follows: the first coordinate indicates the distance between the point and the axis of symmetry of the pot on the axis $\mathrm{X}$ and it represents the diameter of the pot, while the second coordinate indicates the height of the point compared to the origin 0 of the axis of symmetry on axis $Z$ (Figure $3 a$ ). Once the drawing of the profile line is made, the three-dimensional generation of the pot is done using the Revolve command of the chosen design software (Figure $3 \mathrm{~b}$ ).

10 BADIU/POPESCU/CENUSA/BUNA/COMES 2014, 21-25.

11 KERN 2001.

12 MELERO/LEON/CONTRERAS/TORRES 2003, 78-81; MELERO/LEON/TORRES 2010, 137-141; KAMPEL/MELERO 2003, 79-88; SHEAR 2008; KENEDA 2009; MARA/ SABLATNIG 2006, 349-357; MARA/ SABLATNIG 2004, 123-133; HALÍŘ 1999.
In other words, the main goal of the parameterization phase is to obtain the two coordinates for each of the 8 inflection points that make up the profile line (spline). Moreover, by changing these two values for each of the eight inflection points located on the NUPBS curve (Non-UniformPolynomial-B-Spline) the entire profile of the vessel can be redefined.

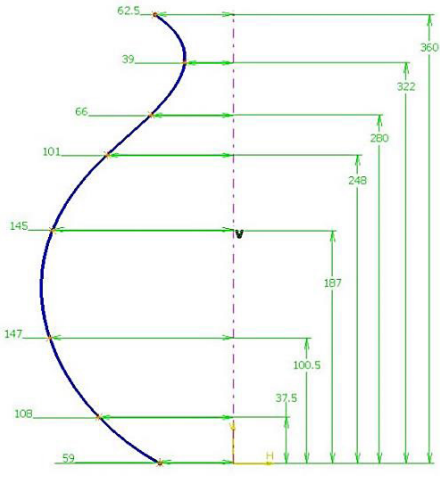

a)

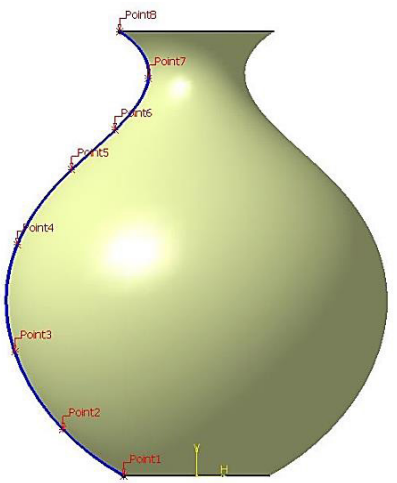

b)
Fig. 3. a) Dimensioning the inflection points in relation to the reference system (axis of symmetry of the pot);

b) the profile of the pot generated using the chosen design software.

The number of eight inflection points is strategically chosen because it offers the possibility of generating profiles for a wide range of pottery families (with a high complexity and precision).

Table 1 illustrates 15 families of pots generated within the case study, along with the set of two coordinates that define the height and diameter of various pots contained in the study.

\section{PROFILES \\ USING MACROS FOR THE GENERATION OF \\ The achievement of the profile and the} parameterization of the eight inflection points is followed by a second step, namely the achievement of a macro (script) that would automatically generate the profiles of the pots. Essentially, a macro consists of grouping a series of functions as a single command with the use of an encrypted language in order to automatically perform a specific requirement (task), in this case the 3D generation of ceramic pottery. The major advantage of a macro is to reduce the time needed to achieve repeatedly the same requirement. Moreover, the use of such an automated repetitive process lowers the incidence of human error and increases process efficiency through standardization, extension of design program capabilities and optimization. Another positive aspect of using a macro is that, although it employs a programming language, the user does not have to be a specialist or to have programming knowledge in order to be able to operate with this concept ${ }^{13}$.

To serve the case study contained in this paper, the generation of the script (Figure 4) was done in the same design software as previously used for the creation and parameterization of the profile line, namely CATIA software, in which this function is found in the toolbar under the Macro section.

13 ROSS 2013, 12. 
Tab. 1. Families of ceramic pots generated within the case study using eight inflection points on the profile line.

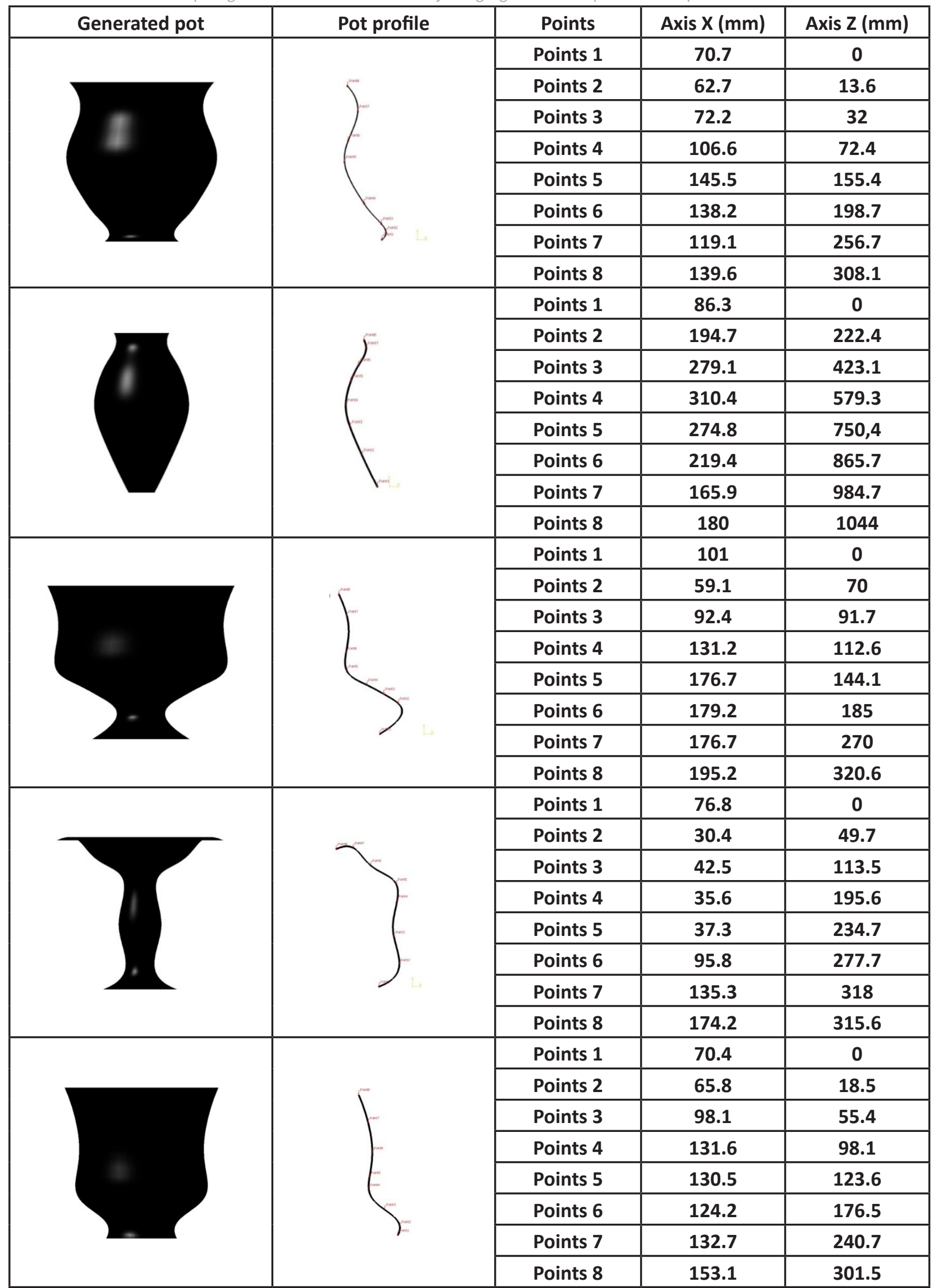




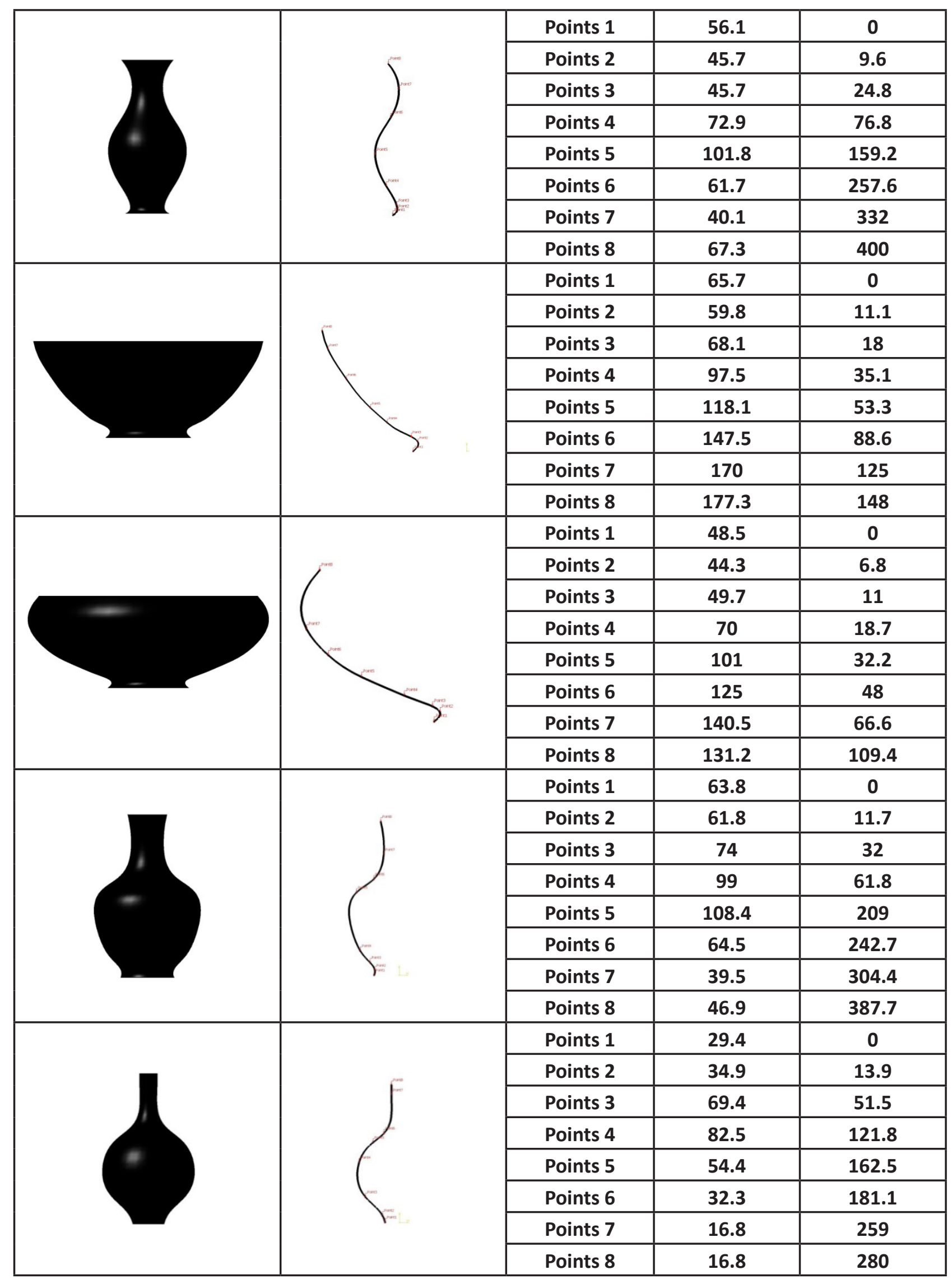




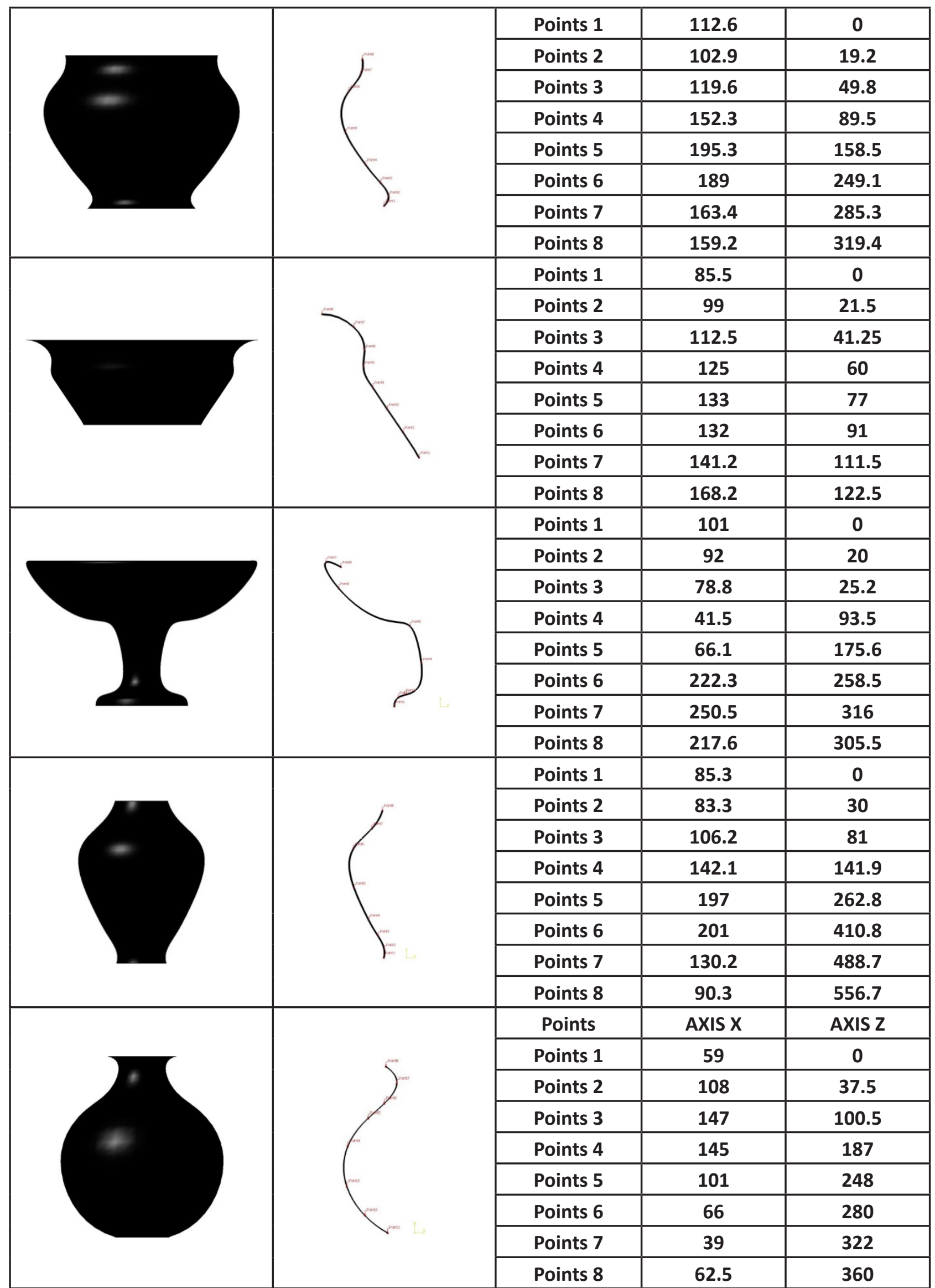




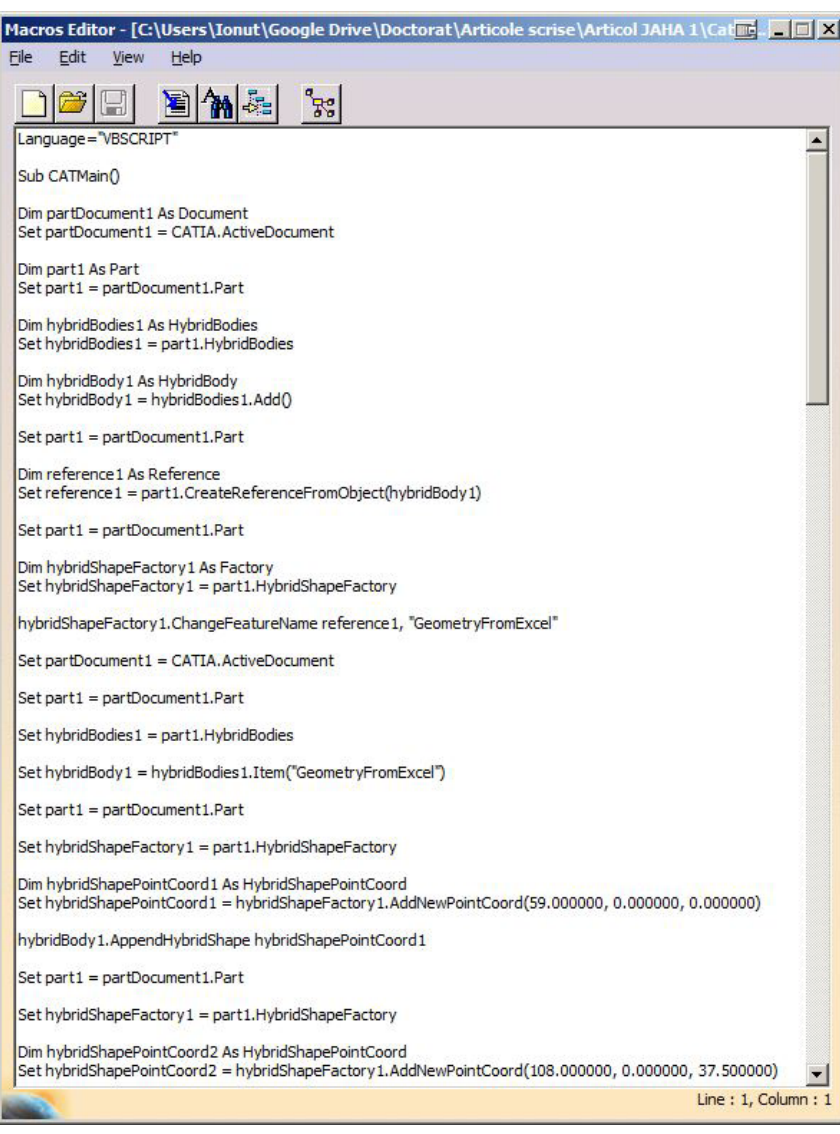

Fig. 4. Macro Editor.

The purpose of this technique is to serve as a support both for historians, archaeologists and restorers, as well as for students who desire a fast, fair and accessible virtual reconstruction of ceramic pots, regardless of their considered knowledge in designing or editing a programming language. Moreover, given that most often restorers do not have the necessary time or financial resources to restore all the objects discovered on site, thus ceramic fragments ending up being stored in warehouses, the proposed methodology aims to be a fast and simple reconstruction tool, regardless of the user's field of work and specialty. Sometimes reconstruction cannot be done because, according to current regulations,

Dim hybridShapePointCoord1 As HybridShapePointCoord

Set hybridShapePointCoord 1 = hybridShapeFactory 1 . AddNewPointCoord $(59.000000,0.000000,0.000000)$

hybridBody 1.AppendHybridShape hybridShapePointCoord1

Set part1 $=$ partDocument1.Part

Set hybridShapeFactory $1=$ part1. HybridShapeFactory

Dim hybridShapePointCoord2 As HybridShapePointCoord

Set hybridShapePointCoord2 = hybridShapeFactory 1.AddNewPointCoord(108.000000, 0.000000, 37.500000)

hybridBody 1. AppendHybridShape hybridShapePointCoord2

Set part1 $=$ partDocument1.Part

Set hybridShapeFactory 1 = part1. HybridShapeFactory

Dim hybridShapePointCoord3 As HybridShapePointCoord

Set hybridShapePointCoord3 $=$ hybridShapeFactory 1. AddNewPointCoord(147.000000, $0.000000,100.500000$ )

Fig. 5. Macro Editor one may only restore objects of which are kept at least $50 \%$ of the original ${ }^{14}$. The best solution to these problems therefore remains the virtual reconstruction of such objects.

By means of the methodology presented, for editing the profile of the pot and thus its shape, restorers have the possibility to modify directly through the script contained in the design software the coordinate values for the two axes, in order to determine the height and diameter of the pot (Figure 5). Such a modification is simple and requires no additional orders.

Below is an excerpt from the script created by the authors in which the first point of inflection on the profile line is generated. From the set comprised of the two geometric coordinates of the inflection point, it can be seen that the point is located at the basis of the pot, and the pot has a diameter of $59 \mathrm{~cm}$.

\section{CONCLUSIONS}

Digitization of ceramic pottery is currently made by various methods of which some are based on photogrammetry, while others are based on laser scanning. Both methods are improving from day to day as a result of ample research aimed at obtaining 3D models with high accuracy. In fact, digitization is primarily aimed to protect the integrity of heritage objects and, at the same time, to ensure the quality in terms of $3 \mathrm{D}$ reconstructions.

Through this paper, the authors have proposed a step forward in terms of profile reconstruction of ancient pottery. The method presented hereby is based on photogrammetry, by which the profile line of the pot is obtained. This line, also known as spline, is set up with eight points of inflection, each of these points being described by a pair of values relative to a reference system defined by the axis of symmetry of the pot. The parameterization of the profile thus constitutes the input to a macro type application (script) contained by the CAD design software. The output of this operation consists in the automatic generation of the three-dimensional shape of the pot. Besides the fact that the generation method through eight points of inflection and macro-type application is easy to use and edit, it also presents the major advantage of shortening the time for the virtual representation of ceramic pottery artifacts.

The main aim of the method is to support the historians, archaeologists, restorers, students and not only, through a quicker and more accessible technique, regardless of the knowledge covered by either of them in programming language and modeling in CAD Software.

Furthermore, the collaboration between the fields of engineering

14 MOLDOVEANU 1999, 345-348. 
Dim point2D1 As Point2D

Set point2D1 = factory2D1. CreatePoint (59.000000, 0.000000)

point2D1.ReportName $=3$

point2D1. Construction $=$ False

Dim constraints1 As Constraints

Set constraints1 = sketch1. Constraints

Dim reference2 As Reference

Set reference2 = part1. CreateReferenceFromObject(point2D1)

Dim reference3 As Reference

Set reference3 $=$ part1. CreateReferenceFromObject(line2D2)

Dim constraint1 As Constraint

Set constraint1 = constraints1.AddBiEltCst(catCstTypeDistance, reference2, reference3)

constraint1. Mode $=$ catCstModeDrivingDimension

Dim length1 As Dimension

Set length1 = constraint1.Dimension

length1.Value $=59.000000$

Dim reference4 As Reference

Set reference4 = part1. CreateReferenceFromObject(point2D1)

Dim reference5 As Reference

Set reference5 = part1. CreateReferenceFromObject(line2D1)

Dim constraint2 As Constraint

Set constraint2 = constraints1.AddBiEltCst(catCstTypeDistance, reference4, reference5)

constraint2. Mode $=$ catCstModeDrivingDimension

Dim length2 As Dimension

Set length2 = constraint2. Dimension

length2.Value $=0.000000$

The second excerpt from the generated script illustrates the application of the Revolve function in order to generate the threedimensional pot. This function is performed with a reference of 360 degrees.

\section{--\#\# command of Revolve function \#\#}

Dim partDocument1 As Document

Set partDocument1 = CATIA.ActiveDocument

Dim part1 As Part

Set part1 = partDocument1. Part

Dim hybridShapeFactory1

As Factory

Set hybridShapeFactory1 = part1. HybridShapeFactory

Dim bodies1 As Bodies

Set bodies1 = part1. Bodies

Dim body1 As Body

Set body1 = bodies1.Item("PartBody")

Dim hybridShapes1 As HybridShapes

Set hybridShapes1 = body1.HybridShapes

Dim hybridShapeSpline1 As HybridShape

Set hybridShapeSpline1 = hybridShapes1.Item(“Spline.1”)

Dim reference1 As Reference

Set reference1 = part1. CreateReferenceFromObject(hybridShapeSpline1)

Dim sketches1 As Sketches

Set sketches1 = body1.Sketches

Dim sketch1 As Sketch

Set sketch1 = sketches1.Item("Sketch.2")

Dim geometricElements1 As GeometricElements

Set geometricElements1 = sketch1.GeometricElements

Dim axis2D1 As GeometricElement

Set axis2D1 = geometricElements1.Item(“AbsoluteAxis")

Dim reference2 As CATBaseDispatch

Set reference2 = axis2D1.GetItem("VDirection")

Dim hybridShapeRevol1 As HybridShapeRevol

Set hybridShapeRevol1 = hybridShapeFactory1.AddNewRevol(reference1, 360.000000, 0.000000, reference2)

body1.InsertHybridShape hybridShapeRevol1

part1.InWorkObject = hybridShapeRevol1

part1.Update

part1.Update 
and archeology is definitely a useful one, since knowledge management of ceramic pottery metadata is an important starting point for digital reconstruction and processing. In addition, the collaboration between these two areas brings more knowledge in terms of research and its further development.

The information resulting from the virtual modeling, together with those provided by databases, facilitate the classical (manual) reconstruction, resulting in a more accurate and rapid restoration of pottery type historical artifacts.

\section{ACKNOWLEDGEMENTS}

This paper is supported by the Sectorial Operational Programme Human Resources Development POSDRU/159/1,5/S/137516 financed from the European Social Fund and by the Romanian Government.

\section{REFERENCES}

\section{BADIU/POPESCU/CENUSA/BUNA/COMES 2014}

Badiu, I./ Popescu, D./ Cenusa, A./ Buna, Z./ Comes, R., Using CAD software to reduce the amount of data in case of digital preservation of the Cultural Heritage, International Conference on Production Research - Regional Conference Africa, Europe and the Middle East, 3rd International Conference on Quality and Innovation in Engineering and Management, 21-25.

BRUNO/BRUNO/DE SENSI/LUCHI/MANCUSO/MUZZUPAPPA 2010

Bruno, F./ Bruno, S./ De Sensi, G./ Luchi, M.L./ Mancuso, S./ Muzzupappa, M., From 3D reconstruction to virtual reality: A complete methodology for digital archaeological exhibition, Journal of Cultural Heritage 11/1, 42-49.

CHOW/CHAN 2009

Chow, S.K./ Chan, K.L., Reconstruction of photorealistic 3D model of ceramic artefacts for interactive virtual exhibition, Journal of Cultural Heritage 10/2, 161-173.

COHEN/LIU/EZGI 2013

Cohen, F./ Liu, Z./ Ezgi, T., Virtual reconstruction of archeological vessels using expert priors and intrinsic differential geometry information, Computers \& Graphics 37/1-2, 41-53.

COMES/BUNA/BADIU 2014

Comes, R./ Buna, Z./ Badiu, I., Creation and preservation of digital Cultural Heritage, Journal of Ancient History and Archeology 1/1, 50-57.

CURSARU HERLEA 2008

CURSARU HERLEA, S.M., Consideraţii privind conservarea şi restaurarea unor vase ceramice medievale timpurii descoperite la Capidava, Studia Universitatis Cibiniensis. Historica 5, 249-262.

GHEORGHIU 2005

Gheorghiu, G., Dacii pe cursul mijlociu al Mureșului (ClujNapoca: Mega), 138-146.

GILBOA/TAL/SHIMSHONI/KOLOMENKIN 2013

Gilboa, A./ Tal, A./ Shimshoni, I./ Kolomenkin, M., Computer-based, automatic recording and illustration of complex archaeological artifacts, Journal of Archaeological Science. 40/2, 1329-1339.

HALÍř 1999

Halír, R., An Automatic Estimation Of The Axis Of Rotation Of Fragments Of Archaeological Pottery: A Multi-Step Model-Based Approach. In: Proceedings of the 17th International Conference in Central Europe on Computer Graphics, Visualization and Interactive Digital Media [Journal of WSCG 17/1-3], 1-7.

ITSKOVICH/TAL 2011

Itskovich, A./ Tal, A., Surface partial matching and application to archaeology, Computers \& Graphics 35/2, 334341.

KAMPEL/MELERO 2003

Kampel, M./ Melero, F.J., Virtual Vessel Reconstruction from a Fragment's Profile. In: Arnold, D.B./ Chalmers, A./ Niccolucci, F. VAST 2003: the 4th International Symposium on Virtual Reality, Archaeology, and Intelligent Cultural Heritage, incorporating 1st EUROGRAPHICS Symposia on Graphics and Cultural Heritage, 79-88.

KAMPEL/SABLATNIG 2003

Kampel, M./ Sablatnig, R., Virtual reconstruction of broken and unbroken pottery. In: Fourth International Conference on 3-D Digital Imaging and Modeling: 3DIM 2003, 6-10 October 2003, Banff, Alberta, Canada: proceedings (Los Alamitos: IEEE Computer Society), 318-325.

KARASIK/SMILANSKY 2008

Karasik, A./ Smilansky, U., 3D scanning technology as a standard archaeological tool for pottery analysis: practice and theory, Journal of Archaeological Science 35/5, 11481168.

\section{KENEDA 2009}

Keneda, A., Application of a low cost laser scanner for archaeology in Japan. In: Stylianidis, E./ Patias, P./ Quintero, M.S. (eds.), CIPA heritage documentation: best practices and applications : series 1, 2007 \& 2009: XXI International Symposium-CIPA 2007, Athens, XXII International Symposium-CIPA 2009, Kyoto.

\section{KERN 2001}

Kern, F., Supplementing laserscanner geometric data with photogrammetric images for modeling, Surveying and Documentation of Historic Buildings - Monuments - Sites Traditional and Modern Methods.

KOUTSOUDIS/ARNAOUTOGLOU/GHAMZAS 2007

Koutsoudis, A./ Arnaoutoglou, F./ Chamzas, C., On 3D reconstruction of the old city of Xanthi. A minimum budget approach to virtual touring based on photogrammetry, Journal of Cultural Heritage 8/1, 26-31.

\section{LAPP/NICOLI 2014}

Lapp, E./ Nicoli, J., Exploring 3D modeling, fingerprint extraction, and other scanning applications for ancient clay oil lamps, Digital Applications in Archaeology and Cultural Heritage 1/2, 34-44.

\section{LIU/POTTMANN/WANG 2006}

Liu, Y./ Pottmann, H./ Wang, W., Constrained 3D Shape Reconstruction Using a Combination of Surface Fitting and Registration, Computer-Aided Design 38/6, 572-583.

MARA/SABLATNIG 2005

Mara, H./ Sablatnig, R., Semiautomatic and automatic profile generation for archaeological fragments. In: Hanbury, A./ Bischof, H. (eds.), Computer vision winter workshop 2005. Proceedings of the 10th Computer Vision Winter Workshop (CVWW 2005), February 2-4, 2005, Zell an der Pram, Upper Austria (Wien: University of Technology), 123-134.

MARA/SABLATNIG 2006

Mara, H./ Sablatnig, R., Determination of Ancient Manufacturing Techniques of Ceramics by 3D Shape Estimation, Interactive Technologies and Sociotechnical Systems Lecture Notes in Computer Science 4270, 349-357.

\section{MARIE/QASRAWI 2005}

Marie, I./ Qasrawi, H., Virtual assembly of pottery 
fragments using moiré surface profile measurements, Journal of Archaeological Science 32/10, 1527-1533.

MELERO/LEON/CONTRERAS/TORRES 2003

Melero, F./ Leon, A./ Contreras, F./ Torres, J.C., A new system for interactive vessel reconstruction and drawing, In: Enter the past: the E-way into the four dimensions of cultural heritage: CAA 2003, Computer Applications and Quantitative Methods in Archaeology : proceedings of the 31st conference, Vienna, Austria, April 2003 [Bar International Series 1227] (Oxford: Archaeopress), 78-81.

MELERO/LEON/TORRES 2010

Melero, F./ Leon, A./ Torres, J.C., Digitalización y reconstrucción de elementos cerámicos arqueológicos de torno, Virtual Archaeology Review 1/2, 137-141.

MOLDOVEANU 1999

Moldoveanu, A., Conservarea preventivă a bunurilor culturale (București).

MONTES/MELERO 2010

Montes, R./ Melero, F.J., A Multimedia 3D Game for Museums. In: Niccolucci, F./ Hermon, S. (eds.), Beyond the artifact : digital interpretation of the past : proceedings of CAA2004, Prato 13-17 April 2004 (Budapest: Archeolingua), 355-358.

OXHOLM/NISHINO 2013

Oxholm, G./ Nishino, K., A flexible approach to reassembling thin artifacts of unknown geometry, Journal of Cultural Heritage 14/1, 51-61.

PAVLIDIS/KOUTSOUDIS/ARNAOUTOGLOU/TSIOUKAS/

\section{CHAMZAS 2007}

Pavlidis, G./ Koutsoudis, A./ Arnaoutoglou, F./ Tsioukas, V./ Chamzas, C., Methods for 3D digitization of Cultural Heritage, Journal of Cultural Heritage 8/1, 93-98.

\section{ROSS 2013}

Ross, E., VB Scripting for CATIA V5, in How to program CATIA macros (unknown place of publication).

\section{SHEAR 2008}

Shear, G.,3DScanning for ProfileAcquisitionandReconstruction of Mayan Ceramics, http://www.thepersistentbeat.com/ Tech-Ceramics files/3D\%20Sherd\%20Paper.pdf (accessed on May 19, 2015).

SMITH/KARASIK/NARAYANAN/OLSON/SMILANSKY/LEVY 2014

Smith, N.G./ Karasik, A./ Narayanan, T./ Olson, E.S./ Smilansky, U./ Levy, T.E., The Pottery Informatics Query Database: A New Method for Mathematic and Quantitative Analyses of Large Regional Ceramic Datasets, Journal of Archaeological Method and Theory 21/1, 212-250.

VILCEANU 2013

Vilceanu, C.B., Aplicații practice in fotogrammetria digitală, http://www.ct.upt.ro/users/Clara-BeatriceVilceanu/ Fotogrammetrie.pdf (accessed on May 19, 2015).

WEISE/WISMER/LEIBE/VAN GOOL 2011

Weise, T./ Wismer, T./ Van Gool, L., Online loop closure for real-time interactive 3D scanning, Computer Vision and Image Understanding 115/5, 635-648. 